International Journal of Basic and Applied Sciences, 1 (4) (2012) 511-515

(C) Science Publishing Corporation

www.sciencepubco.com/index.php/IJBAS

\title{
A Model for the HyperKähler Metrics Building Program
}

\author{
A. El Boukili, M.B. Sedra and A. Zemate \\ Université Ibn Tofail, Faculté des Sciences, \\ Département de Physique, LHESIR, Kénitra, Morocco \\ E-mail: msedra@ictp.it
}

\begin{abstract}
It's by now well known that the harmonic superspace provides a strong framework for constructing general hyperKähler metrics. Original results in this issue are given by the Taub-Nut and Egushi-Hanson metrics exhibiting both $U(1)$ Pauli-Gursey isomertie as shown by Gibbon et all in [1]. It's also shown that there exist series of potential depending explicitly on the harmonics variables $u^{ \pm}$on the sphere $S^{2}$. Based on these knowledge and on previous contributions to the program of metrics building $[2,3]$, we contribute by presenting a special hyperKähler potential $H^{+4}$ leading to an $\alpha$-parametrized Liouville equation on the harmonic superspace.
\end{abstract}

Keywords: Harmonic superspace, HyperKähler metrics, Liouville model, Supersymmetry

\section{General Setting}

The problem of hyperKähler metrics building is an interesting question of hyperKähler geometry that can be nicely solved in the harmonic superspace $(H S)[4,5,2]$ if one knows how to solve the following nonlinear differential equations on the sphere $S^{2}$ :

$$
\begin{gathered}
\partial^{++} q^{+}-\partial^{++}\left(\frac{\partial V^{4+}}{\partial\left(\partial^{++} \bar{q}^{+}\right)}\right)+\frac{\partial V^{4+}}{\partial \bar{q}^{+}}=0 \\
\partial^{++} \bar{q}^{++}+\partial^{++}\left(\frac{\partial V^{4+}}{\partial\left(\partial^{++} \bar{q}^{+}\right)}\right)-\frac{\partial V^{4+}}{\partial q^{+}}=0
\end{gathered}
$$


where $q^{+}=q^{+}\left(z, \bar{z}, u^{ \pm}\right)$and its conjugates $\bar{q}^{+}=q^{+}\left(z, \bar{z}, u^{ \pm}\right)$are complex fields defined globally on $\mathbb{R}^{2} \otimes S^{2} \approx \mathbb{C} \otimes S^{2}$, respectively, parametrized by the local analytic coordinates $z, \bar{z}$ and the harmonic variables $u^{ \pm}$. The symbol $\partial^{++}=u^{+i} \partial / \partial u^{-i}$ stands for the harmonic derivative and $V^{4+}=V^{4+}(q, u)$ is an interacting potential depending in general on $q^{+}, \bar{q}^{+}$, their derivatives and the $u^{ \pm}$'s. Note that the fields $q^{+}$and $\bar{q}^{+}$may be expanded in powers of harmonic variables (for the bosonic part) preserving the total $U(1)$ charge, in each term of the expansion as shown here below:

$$
q^{+}(z, \bar{z}, u)=u_{i}^{+} \varphi^{i}(z, \bar{z})+u_{i}^{+} u_{j}^{+} u_{k}^{-} \varphi^{(i j k)}(z, \bar{z})+\ldots
$$

(2), which fix the $u$-dependence of the $q^{+}$'s, is in fact the pure bosonic projection of a two dimensional $N=4$ supersymmetric HS superfield equation of motion. The remaining equations carry the spinor contributions. They describe among others the space time dynamics of the physical degrees of freedom, namely the four bosons $\varphi^{i}\left(z_{,}^{-} z\right) ; i=1,2$ and their $D=2 N=4$ supersymmetric partners, see for example [6].

An equivalent way of writing (2) is to use the Howe-Stelle-Townsend (HST) realization of the $D=2 N=4$ hypermultiplet $\left(0^{4},(1 / 2)^{4}\right)[7]$. In this representation, that we can write

$$
\partial^{++2} \omega-\partial^{++}\left[\frac{\partial H^{4+}}{\partial\left(\partial^{++} \omega\right)}\right]+\frac{\partial H^{4+}}{\partial \omega}=0,
$$

where $\omega=\omega(z, \bar{z}, u)$ is a real field defined on $\mathbb{C} \otimes S^{2}$ and whose leading terms of its harmonic expansion read as:

$$
\omega(z, \bar{z}, u)=u_{i}^{+} u_{j}^{-} f^{i j}(z, \bar{z})+u_{i}^{+} u_{j}^{+} u_{k}^{-} u_{\ell}^{-} g^{i j k \ell}(z, \bar{z})+\ldots
$$

Similary as for (1), the interacting potential $H^{4+}$ depends in general on $\omega$, its derivatives and on the harmonics. In the remarkable case where the potentials $V^{4+}$ and $H^{4+}$ do not depend on the derivatives of the fields $q^{+}$and $\omega,(1)$ reduce to

$$
\begin{aligned}
& \partial^{++} q^{+}+\frac{\partial V^{4+}}{\partial q^{+}}=0 \\
& \partial^{++2} \omega+\frac{\partial H^{4+}}{\partial \omega}=0 .
\end{aligned}
$$

Remark that the solutions of these equations depend naturally on the potentials $V^{4+}$ and $H^{4+}$ and then the finding of these solutions is not an easy question. There are only few examples that had been solved exactly.

\section{The first model:}

Is the Taub-Nut model leading to the well-known Taub-Nut metric of the four dimensional euclidean gravity. Its potential $V^{4+}\left(q^{+}, \bar{q}^{+}\right)$is given by [5] 


$$
V^{4+}=\frac{\lambda}{2}\left(q^{+} \bar{q}^{+}\right)^{2}
$$

where $\lambda$ is a real coupling constant. According to this potential, the equation of motion is

$$
\partial^{++} q^{+}+\lambda\left(q^{+} \bar{q}^{+}\right) \bar{q}^{+}=0
$$

whose solution reads as [5]

$$
q^{+}(z, \bar{z}, u)=u_{i}^{+} \varphi^{i}(z, \bar{z}) \exp -\lambda\left(u_{k}^{+} u_{\ell}^{-} \varphi^{k} \bar{\varphi}^{\ell}\right) .
$$

Note that the knowledge of this solution is an important step towards identifying the metric of the manifold parametrized by the bosonic fields $\varphi^{i}(z, \bar{z})$ and $\bar{\varphi}^{i}(z, \bar{z})$ of the $D=2 N=4$ supersymmetric nonlinear Taub-Nut $\sigma-$ model. The latter possesses an action whose bosonic part reads as ( see [2] and references therein)

$$
S_{B}^{T N}=-\frac{1}{2} \int d z d \bar{z}\left(g_{i j} \partial_{z} \varphi^{i} \partial_{\bar{z}} \varphi^{j}+\bar{g}^{i j} \partial_{z} \bar{\varphi}_{i} \partial_{\bar{z}} \bar{\varphi}_{j}+2 h_{j}^{i} \partial_{z} \varphi^{j} \partial_{\bar{z}} \bar{\varphi}_{i}\right)
$$

Moreover, using the HST representation of the $D=2 N=4$ hypermultiplet, (6) may be rewritten as

$$
\left[\partial^{++}+\lambda \frac{\omega \overleftrightarrow{\partial}^{++} \bar{\omega}}{(1+2 \omega \bar{\omega})}\right]^{2} \cdot \omega=0,
$$

where $\bar{\omega}$ is the complex conjugate of $\omega$ and $\omega \overleftrightarrow{\partial} \overleftrightarrow{\partial}^{++} \bar{\omega}=\omega \partial \bar{\omega}-\partial \omega \cdot \bar{\omega}$. Here also, this equation is exactely solvable. The solution reads as

$$
\omega(z, \bar{z}, u)=u_{i}^{+} u_{j}^{-} f^{i j}(z, \bar{z}) \exp (-i \lambda \beta)
$$

where

$$
\beta=u_{k}^{+} u_{\ell}^{-}\left[f^{0} \bar{f}^{(k \ell)}-\bar{f}^{0} f^{(i j)}+\epsilon_{r s} f^{(k s)} \bar{f}^{(\ell r)}\right] .
$$

The second model:

Is the Eguchi-Hanson model whose potential reads as [5]

$$
H^{4+}(\omega)=\left[\begin{array}{lll}
u_{i}^{+} & u_{j}^{+} \xi^{(i j)}
\end{array}\right]^{2} / \omega^{2},
$$

where $\xi^{i j}$ is an $S U(2)$ real constant triplet.

\section{Solvable Models}

We focus our attention on (3) and look for potentials leading to exact solutions of this equation. The idea is based on suggesting new plausible integrable equations by proceeding by formal analogy with the known integrable two dimensional conformal Liouville equation $[2,3]$. 


\subsection{The HS Liouville Potential}

$$
H^{4+}(\omega, u)=-\frac{1}{2}\left(\frac{\xi^{++}}{\lambda}\right)^{2} \exp 2 \lambda \omega
$$

this result is established in [2] and lead to extract important physical and mathematical properties. We derived the following non linear differential equation of motion

$$
\lambda \partial^{++2} \omega-\xi^{++2} \exp 2 \lambda \omega=0
$$

defining the integrable Liouville field theory. The explicit solution of this nonlinear differential equation reads as

$$
\xi^{++} \exp \lambda \omega=\frac{u_{i}^{+} u_{j}^{+} f^{i j}(z, \bar{z})}{1-u_{k}^{+} u_{\ell}^{-} f^{k \ell}(z, \bar{z})} .
$$

Here we showed, among others, that the integrability of this model is a consequence of the existence of a symmetry generated by the following conserved current

$$
\begin{aligned}
t^{4+} & =\left(\partial^{++} \omega\right)^{2}-\frac{1}{\lambda} \partial^{++2} \omega \\
\partial^{++} t^{4+} & =0 .
\end{aligned}
$$

This representation of the current $t^{4+}$, which in some sense resembles to the Liouville current, can also be obtained by using the field theoretical method or again with the help of an extended Miura transformation which reads, in the general situation, as

$$
\left(\partial^{++n}-W^{2 n+}\right)=\prod_{j=1}^{n}\left(\partial^{++}-V_{j}^{++}\right)
$$

where the fields $V_{j}^{++}, j=1, \ldots, n$, obeys the traceless condition namely

$$
\sum_{j=1}^{n} V_{j}^{++}=0
$$

\subsection{A $\partial^{++} \omega-$ Dependent Potential}

In this section, devoted to a presentation of a new result, we assume that the potential in (3) depends also on the harmonic derivative of the field $\omega$. We assume then the following

$$
H^{4+}\left(\omega, \partial^{++} \omega, u\right)=-\frac{1}{2}\left(\frac{\xi^{++}}{\lambda}\right)^{2} \exp 2 \lambda \omega+\alpha\left(\partial^{++} \omega\right)^{2}
$$


shown easily to give rise to the following non linear differential harmonic equation of motion

$$
\lambda(1-2 \alpha) \partial^{++2} \omega-\xi^{++2} \exp 2 \lambda \omega=0
$$

This equation is similar to the previous HS $2 d$-Liouville field equation of motion modulo a factor term namely $\lambda(1-2 \alpha)$. Setting $\alpha=0$ one recovers the Liouville equation corresponding to the case where the interacting potential $H^{4+}$ is independent of the derivative of $\omega$. We have to underline the singularity of the value $\alpha=\frac{1}{2}$, a problem that we can overcome by making the following rescaling

$$
\xi^{++} \rightarrow \sqrt{1-2 \alpha} \xi^{++}
$$

for $\alpha \neq \frac{1}{2}$. Using this, we can then conclude that both the interacting potential $H^{4+}(\omega, u)$ and $H^{4+}\left(\omega, \partial^{++} \omega, u\right)$ drive us to the same HS Liouville equation of motion

$$
\lambda \partial^{++2} \omega-\xi^{++2} \exp 2 \lambda \omega=0 .
$$

We conclude then that we can associate to the $\alpha$-HS Liouville equation of motion the same stress energy momentum tensor $t^{4+}$. This $\alpha$-HS parametrization deserves more discussion, this task will be reexamined in our next work.

\section{References}

[1] G. Gibbons and S. Hawking, Comm.Math.Phys 66 (1979) 291;

G. Gibbons, P. Rubback, Comm.Math.Phys 115 (1988) 267;

G. Gibbons, D. Olivier, P. Rubback, G. Valent, Nucl.Phys.B 296 (1988) 679 .

[2] E.H. Saidi and M.B. Sedra, Mod.Phys. Lette. A V9 (1994) 3163

[3] M.B. Sedra, Nucl.Phys.B 513 (1998) 709.

[4] A. Galperin, E. Ivanov, V. Ogievetsky, E. Sokatchev, JETP, Lett. 40 (1984) 912 ,

A. Galperin, E. Ivanov, S. Lalitzin, V. Ogievetsky, E. Sokatchev, Class.Quant.Grav. 1 (1984) 469.

[5] A. Galperin, E. Ivanov, V. Ogievetsky, P.K. Townsend, Class.Quant.Grav.3 (1984) 625,

A. Galperin, E. Ivanov, V. Ogievetsky, E. Sokatchev, Comm.Math.Phys 103 (1986) 515.

[6] E.H. Saidi and M.B. Sedra, Int. J. Mod.Phys. A 9 (1994) 891,

E.H. Saidi and M.B. Sedra, Class. Quant. Grav. V10 (1993) 1937.

[7] P. How, K. Stelle and P.K. Townsend,Nucl.Phys.B 214(1983) 319. 